3

4

5

6

7

\section{8}

9

10

\title{
Very small spontaneous structural mutation rate in green algae
}

\author{
Matéo Léger-Pigout ${ }^{1}$, Marc Krasovec ${ }^{1 *}$ \\ ${ }^{1}$ CNRS, Biologie Intégrative des Organismes Marins (BIOM), Observatoire Océanologique, \\ Banyuls sur mer, 66650, France \\ *marc.krasovec@obs-banyuls.fr
}

\section{RUNNING TITLE}

Structural mutation rate in green algae

\section{KEY WORDS}

mutation rate, structural mutation, mutation accumulation, phytoplankton

\section{ABSTRACT}

Structural mutations are very important in evolution and led to major innovations, but our knowledge of the spontaneous structural mutation rate is very limited. We so cannot have a complete view of the adaptive potential of species and new variant in a population without addressing this lack. Here, we used Illumina genomic data of mutation accumulation experiments from five green algae species and measured very low structural mutation rate, between $5 \times 10^{-12}$ to $2 \times 10^{-11}$ mutations per site, at least ten times lower than the nucleotide mutation rate in these species. Such low rates of structural mutations are typical of species with large effective population sizes, and suggests a very strong selection toward a small structural mutation rate. Last, we show that the rate of true positive detection of de novo structural mutations is variant types dependent and globally poor, highlighting the necessity of long read sequencing in future mutation accumulation studies. 


\section{INTRODUCTION}

Spontaneous mutations renew the standing genetic variation and permit the adaptation of species through natural selection. The spontaneous mutation rate $\mu$ is so one of the most important parameters to know in evolution. Since decades biologists tried to measure it using mutation accumulation (MA) experiment (Halligan \& Keightley, 2009), indirectly with a phenotypic approach by following fitness variation of MA lines (Mukai, 1964), and then directly by sequencing (Lynch et al., 2016; Katju \& Bergthorsson, 2019). Our knowledge made significant progresses the last fifteen years, particularly on the nucleotide mutation rate $\mu_{b s}$ (Lynch et al., 2016; Katju \& Bergthorsson, 2019) and short insertion-deletion (indel) mutation rate $\mu_{i d}$ (Sung et al., 2016). We observe a large variation of $\mu_{b s}$ and $\mu_{\text {id }}$ between species, from $\sim 1 \times 10^{-11}$ to $\sim 1 \times 10^{-8}$ in ciliates and humans, respectively. This variation is in part explained by the drift barrier hypothesis (Sung et al., 2012) where high effective population size $\left(N_{e}\right)$ species reaches lower $\mu$ than species with small $N_{e}$. This is due to the strength of selection that pushes harder toward a small $\mu$ (and so limiting the mutation load) in large population where drift is limited. This hypothesis is the most accepted to explain the mutation rate variation between species, even if other factors impact it but with smaller effect such as GC content (Krasovec et al., 2017), genome size, generation time or metabolic rate (Martin \& Palumbi, 1993; Mooers \& Harvey, 1994; Thomas et al., 2010; Weller \& Wu, 2015). Both $\mu_{b s}$ and $\mu_{i d}$ depends so mostly of $N_{e}$, but our knowledge about the spontaneous structural mutation rate $\mu_{\text {st }}$ is very poor (Press et al., 2019; Ho \& Schaack, 2021) and available in only few models (see Table 3 of Katju and Bergthorsson 2019 for copy number variant for example). Structural mutations, defined here as duplications, large insertions-deletions, inversions or chromosome rearrangements, may have stronger phenotypic effect than nucleotide and short indels mutations because they impact a larger portion of the genome. Thus, the pressure toward a small $\mu_{s t}$ should be stronger than for $\mu_{b s}$ and $\mu_{i d}$. In addition, it is impossible to have a complete few of new variants and adaptive potential of a species with limited knowledges of $\mu_{\text {st. }}$ Structural mutations led to major evolutive innovations, for example the supergenes following inversions. In birds, this process saw the emergence of different morphotypes in a same species with different colours, behaviours and feather shapes (Küpper et al., 2016; Tuttle et al., 2016). Here, we measured $\mu_{\text {st }}$ for five species of green algae using mutation accumulation experiment Illumina reads from previous publications (Krasovec et al., 2016, 2017, 2018).

\section{(1)}




\section{MATERIALS AND METHODES}

Raw reads (150 and 300 bp paired-end) were mapped to the reference genomes with bwa mem (Li \& Durbin, 2010), then bam files were treated with samtools (Li et al., 2009) and structural variant calling was done with delly (Rausch et al., 2012), svaba (Wala et al., 2018) and lumpy (Layer et al., 2014) with standard parameters (excepted ploidy at 1). For svaba, we used the option $-n$ to input the $T_{0}$ genome bam file as control. VCF files were then sorted with several threshold to increase the rate of true de novo mutation identification: the mutated site must be covered by 10 in the concerned MA line and the T0 genome; the mapping quality of the site must be higher than 20; the variant must be present in only one MA line, expected for few variants present in two or three MA lines because of non-independent lines (in that case common mutations and generations were count as one); the variant must totalize all the coverage of the site; last, a part of the structural variants appeared in only one MA line, but were not count as a mutation candidate because other lines had same variants but at different positions within the confidence interval of start-end positions. De novo structural mutations candidates were then checked by PCR and Sanger sequencing when possible and by coverage with samtools depth.

\section{RESULTS AND DISCUSSION}

\section{False - true positive rate of structural de novo mutation detection}

The total number of de novo structural mutations candidates was 26 considering the 5 species and the 3 software (Table 1), but PCR and Sanger sequencing check considerably reduced this number to 1 , given a very low true positive rate of $4 \%$. From Sanger sequencing, the 8 tested breaks from svaba and delly were false, so we considered all other non-tested breaks (10) as false. The other variants were deletions (5) and duplications (3), all larger than $300 \mathrm{nt}$ apart one deletion of $215 \mathrm{pb}$. According to Wala et al (Wala et al., 2018), the positive rates of deletions larger than $300 \mathrm{bp}$ are $85 \%$ for svaba and delly, and $95 \%$ for lumpy; but for insertion/duplication, the validation rates decrease to $37 \%, 24 \%$ and $29 \%$. Here, the positive rate of deletion is $20 \%$, substantially lower than previously reported. Regarding duplications, the 3 candidates were false according to PCR and coverage check (two candidates were not verified by PCR). The true positive rate of duplications is so $0 \%$ here, but with only 3 candidates this rate should be taken with caution. Overall, these results give an idea of the difficulties to identify structural de novo mutations with good accuracy particularly with short reads. This result also shows that structural mutations are very rare in these types of experiments, making any statistics on structural mutations spectrum and distribution not often possible. In yeast, regarding duplications, only three events were identified in a previous study with 145 lines maintained for about 2,000 generation each (Zhu et al., 2014). 


\section{$\mu_{s t}$ is more than 10 time lower than $\mu_{b s}$ and $\mu_{i d}$}

111 The single de novo mutation validated by PCR is a deletion of 1,382 bp identified by lumpy in

112 Ostreoccocus mediterraneus on a very variable chromosome in this species, named outlier 113 chromosome (Yau et al., 2020). The mutated region is intergenic and the deleted sequence 114 appears elsewhere in the genome (Table S1). This makes this deleted sequence a possible repeat region resulting from transposable elements, commonly known to be implicated if this type of mutation. This single mutation gives a very low $\mu_{\text {st }}$ for the 5 species, between $5 \times 10^{-12}$ and $2 \times 10^{-11}$ structural mutation per site per haploid genome per generation (Figure 1). It is at least ten times lower than $\mu_{b s}$ and $\mu_{i d}$ in these species. Taking all species together, $\mu_{s t}=2 \times 10^{-12}$. $\mu_{s t}$ in green algae are so among the lower known $\mu$ in the tree of life, similar to $\mu_{b s}$ in Paramecium (Long et al., 2018) and Dictostelium (Kucukyildirim et al., 2020). The selection pressure toward a low $\mu$ in Paramecium species is linked to their very high $N_{e}$ of $\sim 100$ million (Sung et al., 2012). In phytoplankton species, only few $N_{e}$ estimations are available but all turn around few millions to 10 million (Blanc-Mathieu et al., 2017; Krasovec et al., 2019, 2020). $\mu_{b s}$ and $\mu_{i d}$ in phytoplankton are at least ten time higher than in Paramecium. This could indicate that the selection pressure on $\mu_{s t}$ in the green algae is very strong and reduces $\mu_{\text {st }}$ to a value that only very high $N_{e}$ species such as Paramecium can reach for $\mu_{b s}$. One of the origins of such strong selection may be the genome structure of green algae studied here. Their genomes are small, very compacted with few introns and small distance between genes. For example, O. tauri has a genome size of $12.5 \mathrm{Mb}$, with $81.6 \%$ of coding sequences, $82 \%$ of genes without introns and an average distance between genes of $254 \mathrm{bp}$. In that case, any structural mutation has high chance to touch at least one gene or its regulatory region, making almost all structural mutations deleterious. We can hypothesis that it is less the case in larger genomes with a longer distance between genes and high proportion of repeats and non-coding sequences. Another explanation of the low structural mutation rate may come from the mutation mechanisms themself. Many mechanisms act directly on nucleotides such as methylation and oxidation and are known to increase $\mu_{b s}$, making it higher than $\mu_{s t}$ or $\mu_{i d}$ just because single sites have more chance to mutate. Last explanation may be a method bias, e.g the short illumina reads are not the best strategy to detect structural variants. Further researches with mutation accumulation experiments coupled with long reads sequencing is the next milestone significantly increase our knowledge on $\mu_{\text {st. }}$ 
bioRxiv preprint doi: https://doi.org/10.1101/2022.02.09.479524; this version posted February 9,2022 . The copyright holder for this preprint (which was not certified by peer review) is the author/funder, who has granted bioRxiv a license to display the preprint in perpetuity. It is made available under aCC-BY 4.0 International license.

147 Table 1. List of de novo structural mutation candidates. PCR-Sanger and Covergae are the two checking 148 method for de novo mutations. Only the deletion of Om21b is true.

\begin{tabular}{|c|c|c|c|c|c|c|}
\hline $\begin{array}{l}\text { MA line } \\
\text { Ot20 }\end{array}$ & $\begin{array}{l}\text { Mutation type } \\
\text { break }\end{array}$ & $\begin{array}{l}\text { Software } \\
\text { delly }\end{array}$ & $\begin{array}{l}\text { Start } \\
\text { NC 014444.2: } 184889\end{array}$ & $\begin{array}{l}\text { End } \\
\text { NC 014431.2: } 783883\end{array}$ & $\begin{array}{l}\text { PCR - Sanger } \\
\text { false }\end{array}$ & $\begin{array}{l}\text { Coverage } \\
\text { na }\end{array}$ \\
\hline Om14 & break & delly & BCC 102-800924: 233 & BCC 102-1186077: 1181287 & false & na \\
\hline Om17 - 18 & break & delly & BCC102-594986: 60163 & BCC102-768578: 224081 & false & na \\
\hline Om1 & break & delly & BCC102_ML_SOC: 68272 & BCC102-397956: 160264 & false & na \\
\hline Ot38 & deletion & delly & NC_014427.2: 430027 & NC_014427.2: 438281 & no check & false \\
\hline Bp8 & deletion & delly & NC_023992.1: 297114 & NC_023992.1: 301342 & false & false \\
\hline Bp20 & duplication & delly & NC_023992.1: 296522 & NC_023992.1: 297150 & false & false \\
\hline Om13 & break & svaba & BCC̄ 102-873724: 213431 & BCC̄102_ML_SOC: 100063 & false & na \\
\hline Ot22 & break & lumpy & NC_014426.2: 181294 & NC_0144̄28: 229429 & no check & na \\
\hline Ot23 & break & lumpy & NC_014426.2: 929056 & NC_014442.2: 399570 & no check & na \\
\hline Ot9 - 10 & break & lumpy & NC_014435.2: 273589 & NC_014439.2: 385664 & no check & na \\
\hline Ot20 - 31 & break & lumpy & NC_014441.2: 17675 & NC_014443.2: 58813 & no check & na \\
\hline Om38 & break & lumpy & BCC̄102-993683: 535232 & BC̄̄102-768578: 224085 & no check & na \\
\hline Om9 - 29d & deletion & lumpy & BCC10̄2-867403: 33955 & BCC102-867403: 34170 & false & false \\
\hline Om31 & deletion & lumpy & BCC102-466838: 274207 & BCC102-466838: 276502 & false & false \\
\hline Om21b & deletion & lumpy & BCC102_ML_SOC: 571078 & BCC102_ML_SOC: 572460 & true & true \\
\hline Om17 & duplication & lumpy & BCC102-806365: 187888 & BCC102-806365: 188243 & no check & false \\
\hline Om32 & duplication & lumpy & BCC102_ML_SOC: 243207 & BCC102_ML_SOC: 251990 & no check & false \\
\hline
\end{tabular}

A

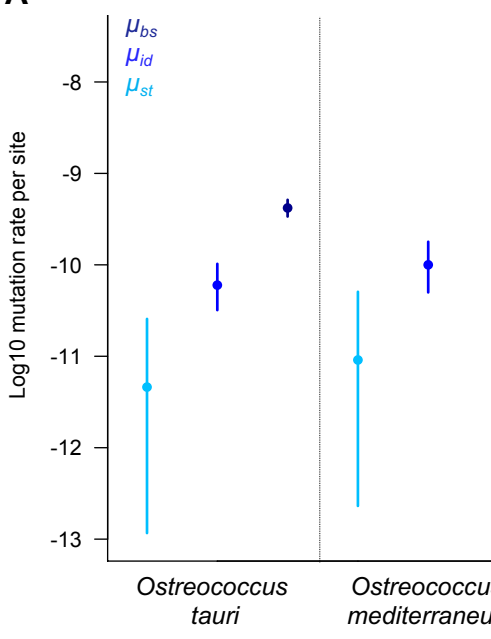

End BCC̄102-1186077: 1181287 BCC102-768578: 224081 BCC102-867403: 392 29715 014428.2:994946 NC 014428: 229429 NC_014442.2: 399570 (14439.2: 385664 BCC102-546026: 350305 no check

Figure 1. A. Spontaneous mutation rate per nucleotide per haploid genome per generation. Black: $\mu_{s t}$, spontaneous structural mutation rate. Dark blue: $\mu_{\text {id }}$, spontaneous short insertion-deletion mutation rate. Blue: $\mu_{b s}$, spontaneous nucleotide mutation rate. Horizontal bars are confidence interval following a Poisson distribution. $\mu_{i d}$ and $\mu_{b s}$ come from previous studies (Krasovec et al., 2017, 2018). $\mu_{s t}$ for $O$. tauri, M. pusilla, B. prasinos et $P$. costavermella are calculated assuming one hypothetical mutation (non has been validated), so the values provided here are the above estimations. B. PCR product migration of one large deletion (1,382 bp in intergenic region) identified in 0 . mediterraneus. The ladder is the 1 kb DNA ladder from Promega. No amplification is observed in the mutation accumulation line Om $21 \mathrm{~b}$ because primers were designed insight the deleted sequence. Om ML is the mother line of the mutation accumulation (T0 genome). 
DATA AVAILABILITY STATEMENT

163

All genomic raw reads used in this study are available on the SRA database in the NCBI under the bioprojects PRJNA531882 (Ostreoccocus tauri, O. mediterraneus, Micromonas pusilla, Bathycoccus prasinos), PRJNA453760 and PRJNA389600 (Picochlorum costavermella).

\section{ACKNOWLEDGEMENTS}

We are grateful to GENOPHY team of the Banyuls sur mer lab for discussions and advices.

\section{AUTHOR CONTRIBUTIONS}

MK designed and managed the study, MLP did bioinformatics analysis and wet lab works, MK wrote the draft of the manuscript, all authors contributed to editing the last manuscript version.

\section{FUNDING}

This work was funded by ANRJCJC-SVSE6-2013-0005.

\section{COMPETING INTERESTS}

The authors declare no conflicts of interest.

\section{BIBLIOGRAPHY}

Blanc-Mathieu, R., Krasovec, M., Hebrard, M., Yau, S., Desgranges, E., Martin, J., et al. 2017. Population genomics of picophytoplankton unveils novel chromosome hypervariability. Sci. Adv. 3: e1700239.

Halligan, D.L. \& Keightley, P.D. 2009. Spontaneous Mutation Accumulation Studies in Evolutionary Genetics. Annu. Rev. Ecol. Evol. Syst. 40: 151-172. Ho, E.K.H. \& Schaack, S. 2021. Intraspecific variation in the rates of mutations causing structural variation in Daphnia magna. Genome Biol. Evol. evab241.

Katju, V. \& Bergthorsson, U. 2019. Old Trade, New Tricks: Insights into the Spontaneous Mutation Process from the Partnering of Classical Mutation Accumulation Experiments with High-Throughput Genomic Approaches. Genome Biol. Evol. 11: 136-165.

Krasovec, M., Eyre-Walker, A., Grimsley, N., Salmeron, C., Pecqueur, D., Piganeau, G., et al. 2016. Fitness Effects of Spontaneous Mutations in Picoeukaryotic Marine Green Algae. G3 GenesGenomesGenetics 6: 2063-2071. Krasovec, M., Eyre-Walker, A., Sanchez-Ferandin, S. \& Piganeau, G. 2017. Spontaneous Mutation Rate in the Smallest Photosynthetic Eukaryotes. Mol. Biol. Evol. 34: 1770-1779. Krasovec, M., Rickaby, R.E.M. \& Filatov, D.A. 2020. Evolution of Mutation Rate in Astronomically Large Phytoplankton Populations. Genome Biol. Evol. 12: 1051-1059. Krasovec, M., Sanchez-Brosseau, S., Grimsley, N. \& Piganeau, G. 2018. Spontaneous mutation rate as a source of diversity for improving desirable traits in cultured microalgae. Algal Res. 35: 85-90. Krasovec, M., Sanchez-Brosseau, S. \& Piganeau, G. 2019. First Estimation of the Spontaneous Mutation Rate in Diatoms. Genome Biol. Evol. 11: 1829-1837. Kucukyildirim, S., Behringer, M., Sung, W., Brock, D.A., Doak, T.G., Mergen, H., et al. 2020. Low Base-Substitution Mutation Rate but High Rate of Slippage Mutations in the Sequence 
Repeat-Rich Genome of Dictyostelium discoideum. G3 Bethesda Md 10: 3445-3452.

Küpper, C., Stocks, M., Risse, J.E., dos Remedios, N., Farrell, L.L., McRae, S.B., et al. 2016. A supergene determines highly divergent male reproductive morphs in the ruff. Nat. Genet. 48: 79-83. Nature Publishing Group. Layer, R.M., Chiang, C., Quinlan, A.R. \& Hall, I.M. 2014. LUMPY: a probabilistic framework for structural variant discovery. Genome Biol. 15: R84. Li, H. \& Durbin, R. 2010. Fast and accurate long-read alignment with Burrows-Wheeler transform. Bioinformatics 26: 589-595.

Li, H., Handsaker, B., Wysoker, A., Fennell, T., Ruan, J., Homer, N., et al. 2009. The Sequence Alignment/Map format and SAMtools. Bioinforma. Oxf. Engl. 25: 2078-2079. Long, H., Doak, T.G. \& Lynch, M. 2018. Limited Mutation-Rate Variation Within the Paramecium aurelia Species Complex. G3 GenesGenomesGenetics 8: 2523-2526. Lynch, M., Ackerman, M.S., Gout, J.-F., Long, H., Sung, W., Thomas, W.K., et al. 2016. Genetic drift, selection and the evolution of the mutation rate. Nat. Rev. Genet. 17: 704-714. Martin, A.P. \& Palumbi, S.R. 1993. Body size, metabolic rate, generation time, and the molecular clock. Proc. Natl. Acad. Sci. U. S. A. 90: 4087-4091.

Mooers, A.O. \& Harvey, P.H. 1994. Metabolic rate, generation time, and the rate of molecular evolution in birds. Mol. Phylogenet. Evol. 3: 344-350.

Mukai, T. 1964. The Genetic Structure of Natural Populations of Drosophila Melanogaster. I. Spontaneous Mutation Rate of Polygenes Controlling Viability. Genetics 50: 1-19.

Press, M.O., Hall, A.N., Morton, E.A. \& Queitsch, C. 2019. Substitutions Are Boring: Some Arguments about Parallel Mutations and High Mutation Rates. Trends Genet. TIG 35: 253264.

Rausch, T., Zichner, T., Schlattl, A., Stütz, A.M., Benes, V. \& Korbel, J.O. 2012. DELLY: structural variant discovery by integrated paired-end and split-read analysis. Bioinformatics 28: i333-i339.

Sung, W., Ackerman, M.S., Dillon, M.M., Platt, T.G., Fuqua, C., Cooper, V.S., et al. 2016. Evolution of the Insertion-Deletion Mutation Rate Across the Tree of Life. G3 Bethesda Md, doi: $10.1534 / g 3.116 .030890$.

Sung, W., Ackerman, M.S., Miller, S.F., Doak, T.G. \& Lynch, M. 2012. Drift-barrier hypothesis and mutation-rate evolution. Proc. Natl. Acad. Sci. U. S. A. 109: 18488-18492.

Thomas, J.A., Welch, J.J., Lanfear, R. \& Bromham, L. 2010. A generation time effect on the rate of molecular evolution in invertebrates. Mol. Biol. Evol. 27: 1173-1180.

Tuttle, E.M., Bergland, A.O., Korody, M.L., Brewer, M.S., Newhouse, D.J., Minx, P., et al. 2016. Divergence and Functional Degradation of a Sex Chromosome-like Supergene. Curr. Biol. CB 26: 344-350.

Wala, J.A., Bandopadhayay, P., Greenwald, N.F., O'Rourke, R., Sharpe, T., Stewart, C., et al. 2018. SvABA: genome-wide detection of structural variants and indels by local assembly. Genome Res. 28: 581-591.

Weller, C. \& Wu, M. 2015. A generation-time effect on the rate of molecular evolution in bacteria. Evol. Int. J. Org. Evol. 69: 643-652.

Yau, S., Krasovec, M., Benites, L.F., Rombauts, S., Groussin, M., Vancaester, E., et al. 2020. Virus-host coexistence in phytoplankton through the genomic lens. Sci. Adv. 6: eaay2587. American Association for the Advancement of Science.

Zhu, Y.O., Siegal, M.L., Hall, D.W. \& Petrov, D.A. 2014. Precise estimates of mutation rate and spectrum in yeast. Proc. Natl. Acad. Sci. U. S. A. 111: E2310-2318. 
bioRxiv preprint doi: https://doi.org/10.1101/2022.02.09.479524; this version posted February 9,2022 . The copyright holder for this preprint (which was not certified by peer review) is the author/funder, who has granted bioRxiv a license to display the preprint in perpetuity. It is made available under aCC-BY 4.0 International license.

258 Table S1. Blastn results of the deleted sequence in Ostrecoccus mediterraneus. The blasted sequence 259 was the sequence amplified by PCR in the MA mother line (404 bp length).

\begin{tabular}{|l|l|l|l|l|l|}
\hline Chromosome & Identity & Length & Start & End & Pvalue \\
\hline BCC102-546026 & 98.916 & 369 & 358951 & 359319 & 0.0 \\
\hline BCC102-546026 & 93.590 & 156 & 356541 & 356696 & $3.17 \mathrm{e}-62$ \\
\hline BCC102-546026 & 89.062 & 128 & 468750 & 468877 & $5.46 \mathrm{e}-40$ \\
\hline BCC102-466838 & 91.617 & 167 & 435797 & 435963 & $9.76 \mathrm{e}-62$ \\
\hline BCC102_ML_SOC & 99.010 & 404 & 321019 & 321422 & 0.0 \\
\hline BCC102_ML_SOC & 98.721 & 391 & 571052 & 571442 & 0.0 \\
\hline BCC102_ML_SOC & 90.678 & 354 & 225814 & 226166 & $2.59 \mathrm{e}-133$ \\
\hline BCC102_ML_SOC & 90.571 & 350 & 257243 & 257591 & $4.34 \mathrm{e}-131$ \\
\hline BCC102_ML_SOC & 90.571 & 350 & 584735 & 585083 & $4.34 \mathrm{e}-131$ \\
\hline BCC102_ML_SOC & 90.299 & 134 & 208824 & 208955 & $2.28 \mathrm{e}-44$ \\
\hline
\end{tabular}

